\title{
Synthesis of arylferrocenylketones
}

\author{
Cristina G. Rodríguez-Cendejas, ${ }^{a}$ Lanny S. Liebeskind ${ }^{1, b}$ and Eduardo Peña-Cabrera* a \\ ${ }^{a}$ Facultad de Química, Universidad de Guanajuato. Col. Noria Alta S/N. Guanajuato, Gto. \\ 36050, Mexico \\ ${ }^{b}$ Emory University, Department of Chemistry, 1515 Pierce Drive, Atlanta, Georgia 30322 USA \\ E-mail: eduardop@quijote.ugto.mx
}

(received 24 May 05; accepted 21 Jun 05; published on the web 30 Jun 05)

\begin{abstract}
The synthesis of a family of mono- and 1,1'-bis-substituted ferrocenylaryl and heteroaryl ketones is described. The key transformation was the Liebeskind-Srögl cross-coupling reaction between ferrocenylthiol esters and boronic acids. The yields ranged from 22 to $99 \%$ in the monosubstituted ferrocenyl derivatives and from 26 to $77 \%$ in the case of the $1,1^{\prime}$-bis-substituted analogues.
\end{abstract}

Keywords: Ferrocenylketones, Liebeskind-Srogl cross-coupling reaction, boronic acids, copper TC, palladium, thiolesters

\section{Introduction}

Ever since its discovery fifty years ago, the chemistry of ferrocene has developed dramatically. ${ }^{2}$ Its remarkable and versatile properties have allowed its use in a wide variety of applications such as homogeneous asymmetric catalysis, organic synthesis, chemical sensors, molecular electronics, and so on. ${ }^{3}$ Ferrocenylketones are an important class of compounds mainly because they can be used as starting materials for derivatives that find important applications such as chemotherapeutic agents. It has been recently reported that ferrocifens $\mathbf{1}$, have antiproliferative properties on both hormone-dependent and hormone-independent breast cell lines. ${ }^{4}$

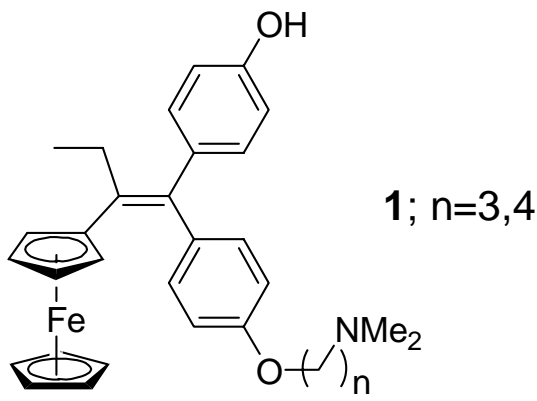


Ferrocenylketones have been employed as well as precursors of arylferrocenylmethanols, compounds that were used as mediators in biosensors. ${ }^{5 a}$ Bildstein and co-workers reported the synthesis of olefin-containing ferrocene derivatives from the corresponding ketones for modeling purposes of molecular electronic devices. ${ }^{6}$ Such olefins have also been used in the synthesis of polymers that are used as coating materials for aerospace applications. ${ }^{7}$ Despite their importance, to the best of our knowledge, just a few ferrocenylmonoketones have been prepared using the Friedel-Crafts reaction, which greatly limits the functional groups that can be attached to the carbonyl group (i.e., the nitrophenyl group). ${ }^{5}$ In this context, Liebeskind and Srögl have developed a Pd-catalyzed, $\mathrm{Cu}(\mathrm{I})$-mediated ketone synthesis that is carried out under mild and neutral conditions. ${ }^{8}$ The Liebeskind-Srögl cross-coupling consists of the reaction of a thiol ester with a boronic acid catalyzed by $\mathrm{Pd}$ and mediated by $\mathrm{Cu}(\mathrm{I})$ under the conditions shown in Scheme 1. 9

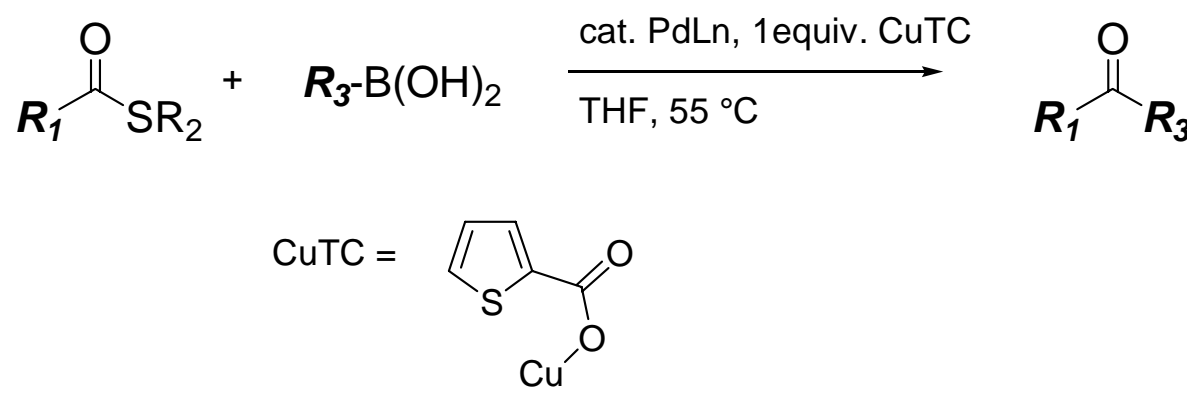

Scheme 1. The Liebeskind-Srögil Cross-Coupling.

In addition to the mild reactions conditions used in this process, the great variety of boronic acids that area commercially available or that can be prepared, ${ }^{10}$ and their non-toxicity, allows for the preparation of a large number of mono- and bisketo derivatives. Moreover, thiol esters can also be efficiently synthesized using methods reported in the literature. ${ }^{11}$ The only practical disadvantage worth mentioning is the rather unpleasant odor of the starting thiols, however, even the "odorless" protocol is known. ${ }^{12}$

Thus, it seemed to us that the Liebeskind-Srögl protocol was suitable for the synthesis of a variety of mono- and bis-ferrocenylketones. The results are reported herein.

\section{Results and Discussion}

The synthesis of the starting materials began with commercially available ferrocenecarboxylic acid 2. Treatment of $\mathbf{2}$ with oxalyl chloride and DMF in chloroform followed by the addition of $p$-methylbenzenthiol in the presence of TEA gave ferrocenylthiol ester 3 in $82 \%$ yield (Figure $1)^{8 b, 13}$ 


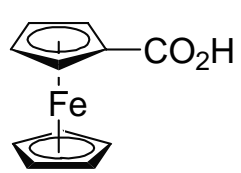

2

\author{
1) $(\mathrm{ClCO})_{2}, \mathrm{CH}_{3} \mathrm{Cl}, 0^{\circ} \mathrm{C}$ \\ 12h, DMF \\ 2) $p-\mathrm{MeC}_{6} \mathrm{H}_{4} \mathrm{SH}, \mathrm{TEA}, \mathrm{C}_{6} \mathrm{H}_{6}$
}

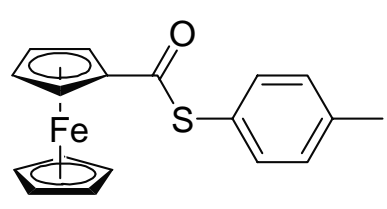

3, $82 \%$

\section{Figure 1}

On the other hand, in order to make the corresponding 1,1'-diubstituted ferrocene analogue, 1, $1^{\prime}$-ferrocenedicarboxylic acid $4^{14}$ was prepared as illustrated in figure 2 in $79 \%$ yield. $^{15}$
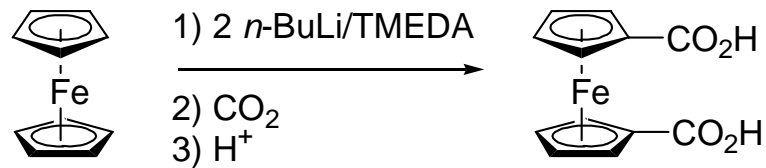

4, $79 \%$

\section{Figure 2}

Afterwards, 4 was reacted under the same conditions as in eq. 1 to give the 1,1'ferrocenebisthiol ester 5 in $85 \%$ yield (figure 3).

$$
\begin{aligned}
& \text { 1) }(\mathrm{ClCO})_{2}, \mathrm{CH}_{3} \mathrm{Cl}, 0{ }^{\circ} \mathrm{C} \\
& 4 \underset{12 \mathrm{~h}, \mathrm{DMF}}{\stackrel{1}{2} \mathrm{p}-\mathrm{MeC}_{6} \mathrm{H}_{4} \mathrm{SH}, \mathrm{TEA}, \mathrm{C}_{6} \mathrm{H}_{6}}
\end{aligned}
$$

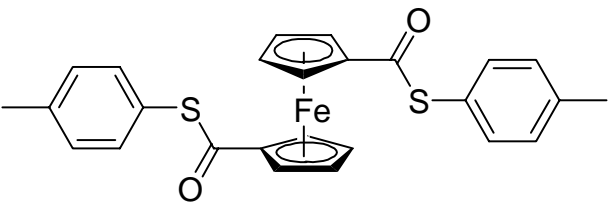

$5,85 \%$

\section{Figure 3}

Both 3 and 5 are stable orange solids which can be stored open to the air and at room temperature without noticeable decomposition. After surveying the optimum reaction conditions, the generality of the cross-couplings of $\mathbf{3}$ and $\mathbf{5}$ was studied (Tables 1 and 2).

Ferrocenyl monoketones. Thiol ester 3 reacted smoothly with a variety of commercially available boronic acids (Table 1). In order to compensate for the dimerization of the boronic acids observed in all reactions, a three-fold excess of these reactants was employed. Varying amounts of starting thiol ester $\mathbf{3}$ were recovered after $18 \mathrm{~h}$. Based on the unreacted $\mathbf{3}$, the yields of the ferrocenylketones were from good to excellent. Simple electron-rich boronic acids reacted efficiently to give the products in excellent yields (entries 1-5). 2-Naphthalene-substituted ketone 
was obtained in lower yield presumably due to the higher steric demand of the substituent $(57 \%$, entry 11). Electron-attracting substituents gave lower yields as well (entries 6 and 9). Heterocyclic derivatives displayed different behavior among them. On the one hand, a very sluggish reaction with benzothiophene-2-boronic acid gave the product in $77 \%$ yield (entry 7 ), however, a large amount of the starting 3 was recovered (64\%). On the other hand, most of the starting 3 reacted with 2-furylboronic acid to produce the product, however, the yield was disappointingly low (22\%, entry 12$)$ suggesting the instability of the product. Cross-coupling reaction with 3-pyridylboronic acid led only to decomposition (entry 8). 1,1' Diferrocenylketone, an important compounds that has been used as precursor for new conducting materials, ${ }^{5 b}$ was synthesized in $69 \%$ yield (entry 10 ).

Table 1. Synthesis of Ferrocenyl Ketones ${ }^{\mathrm{a}}$

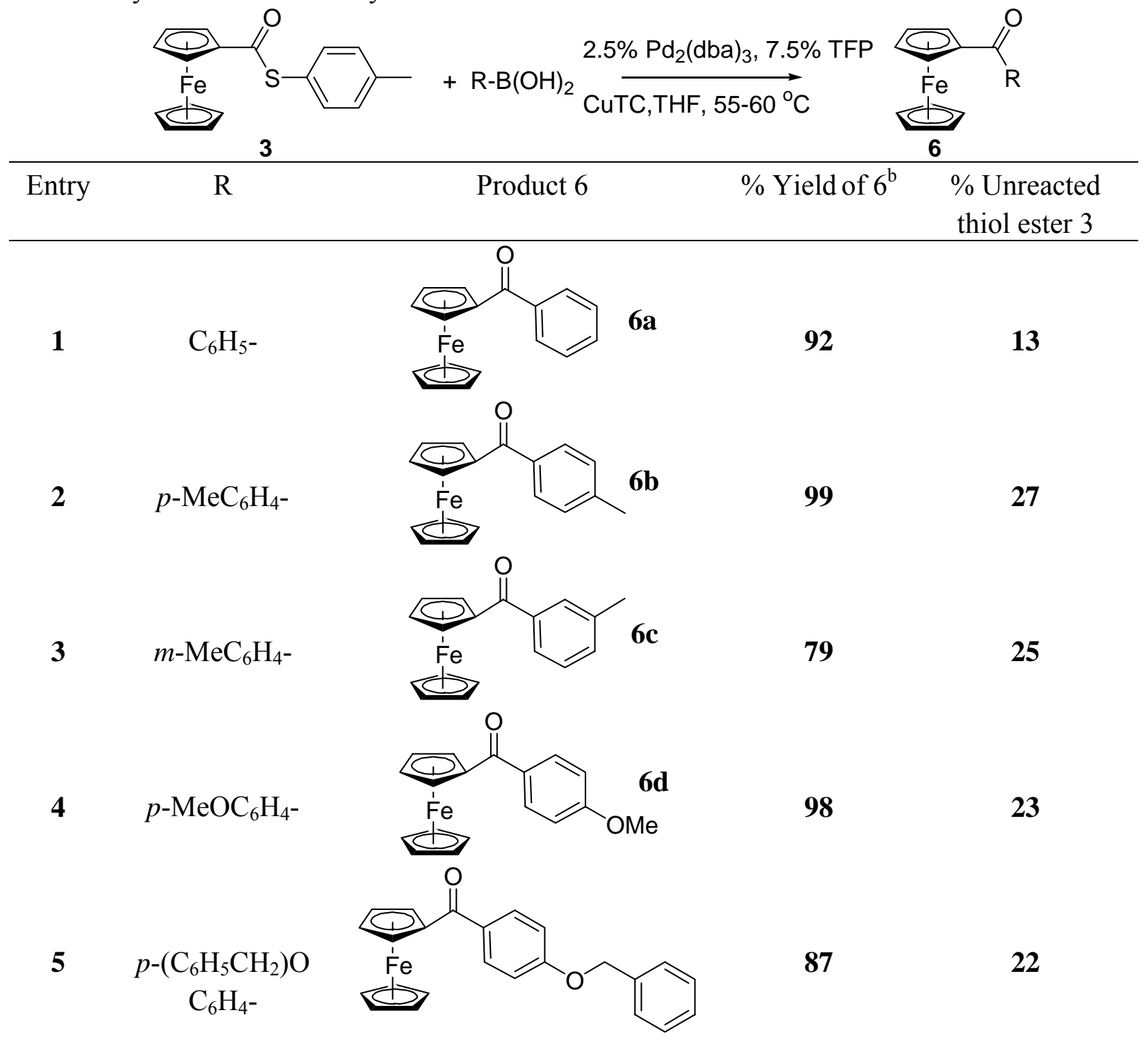


Table 1. Continued

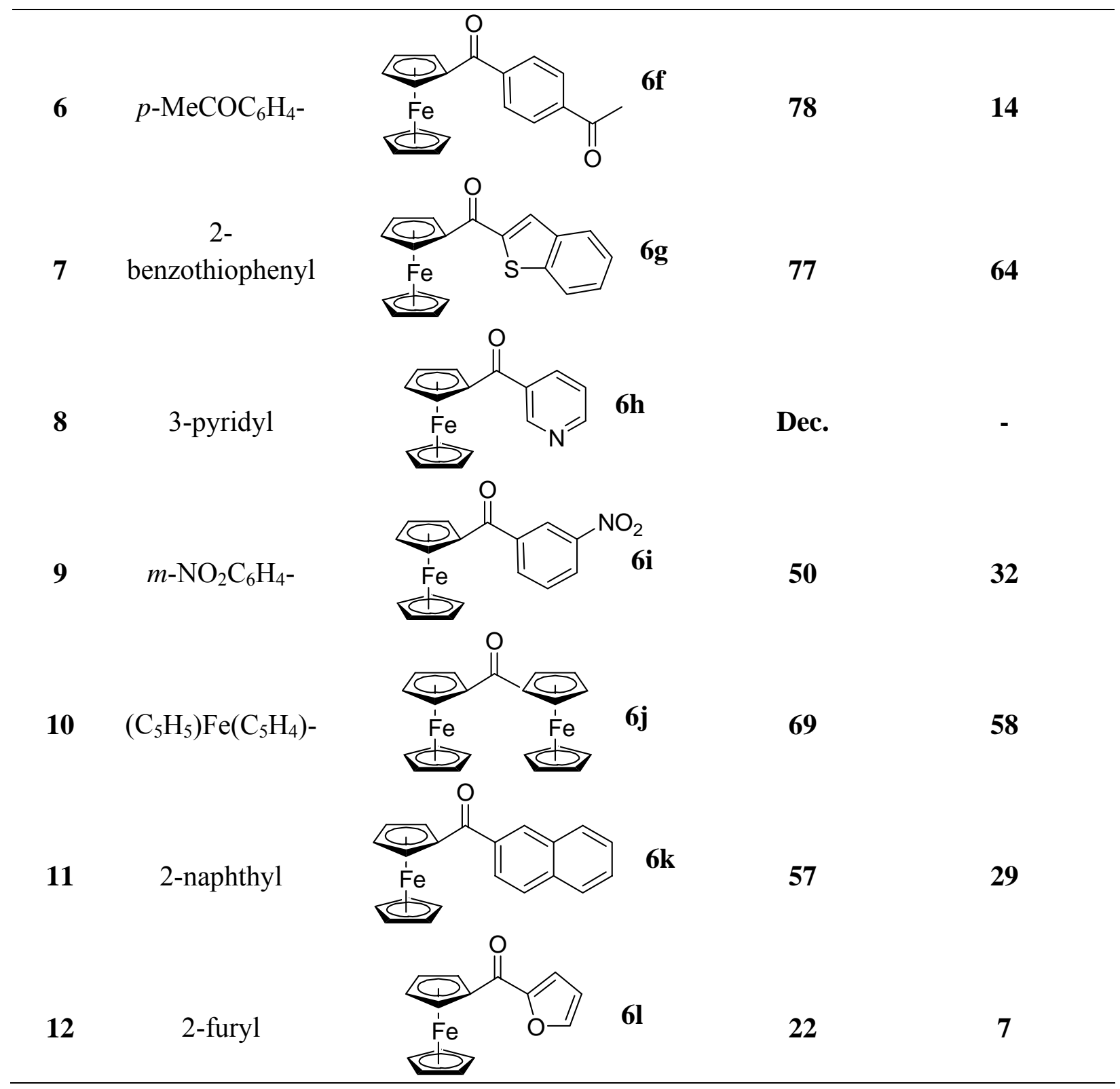

${ }^{a}$ Reaction conditions: thiol ester ( 1 equiv.), $\mathrm{Ar}-\mathrm{B}(\mathrm{OH})_{2}$ (3 equiv.), $\mathrm{Pd}_{2}(\mathrm{dba})_{3} 2.5 \mathrm{~mol} \%$, TFP (tri2 -furylphosphine) $7.5 \mathrm{~mol} \%$, CuTC (4 equiv.), THF, $55^{\circ} \mathrm{C}, 18 \mathrm{~h}$.

${ }^{\mathrm{b}}$ Isolated yields based upon reacted thiol ester.

1,1'-Ferrocenyl diketones. Dithiol ester 5 was reacted with a number of boronic acids with different substitution pattern (Table 2). As in the case of the monosubstituted ferrocene derivatives, a three-fold excess of the boronic acids was necessary to obtain acceptable yields. 
Table 2. Synthesis of 1,1'-Ferrocenyldiketones ${ }^{\mathrm{a}}$
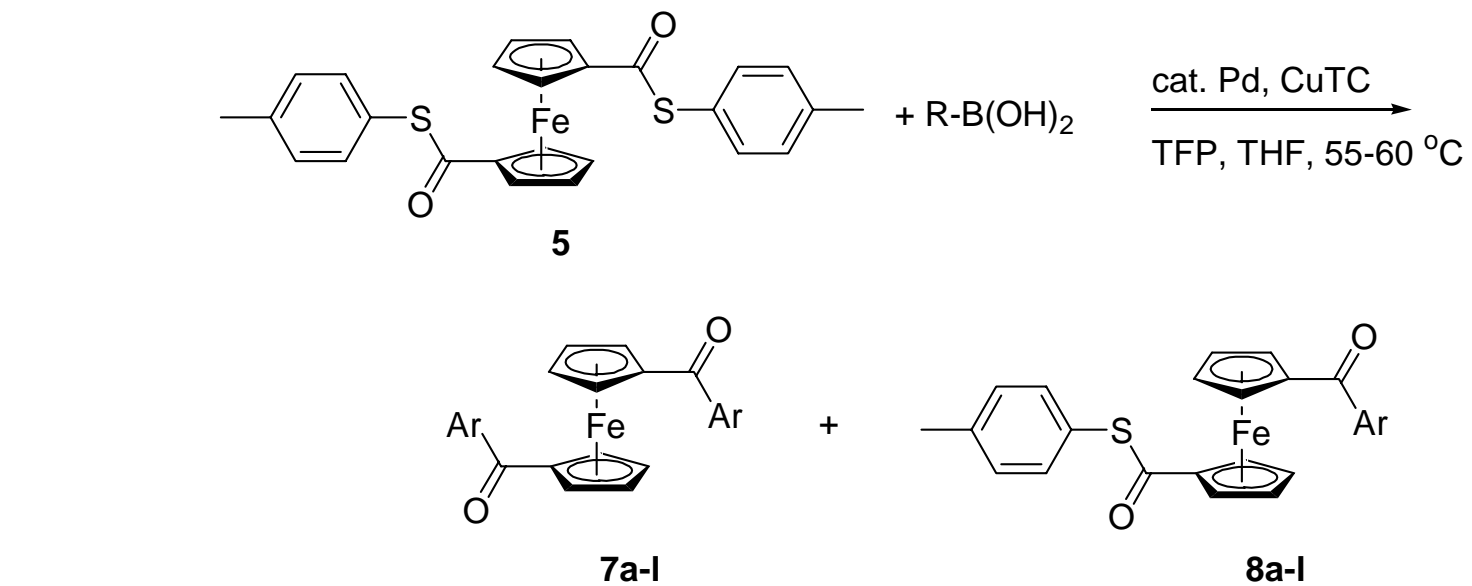

2


Table 2. Continued

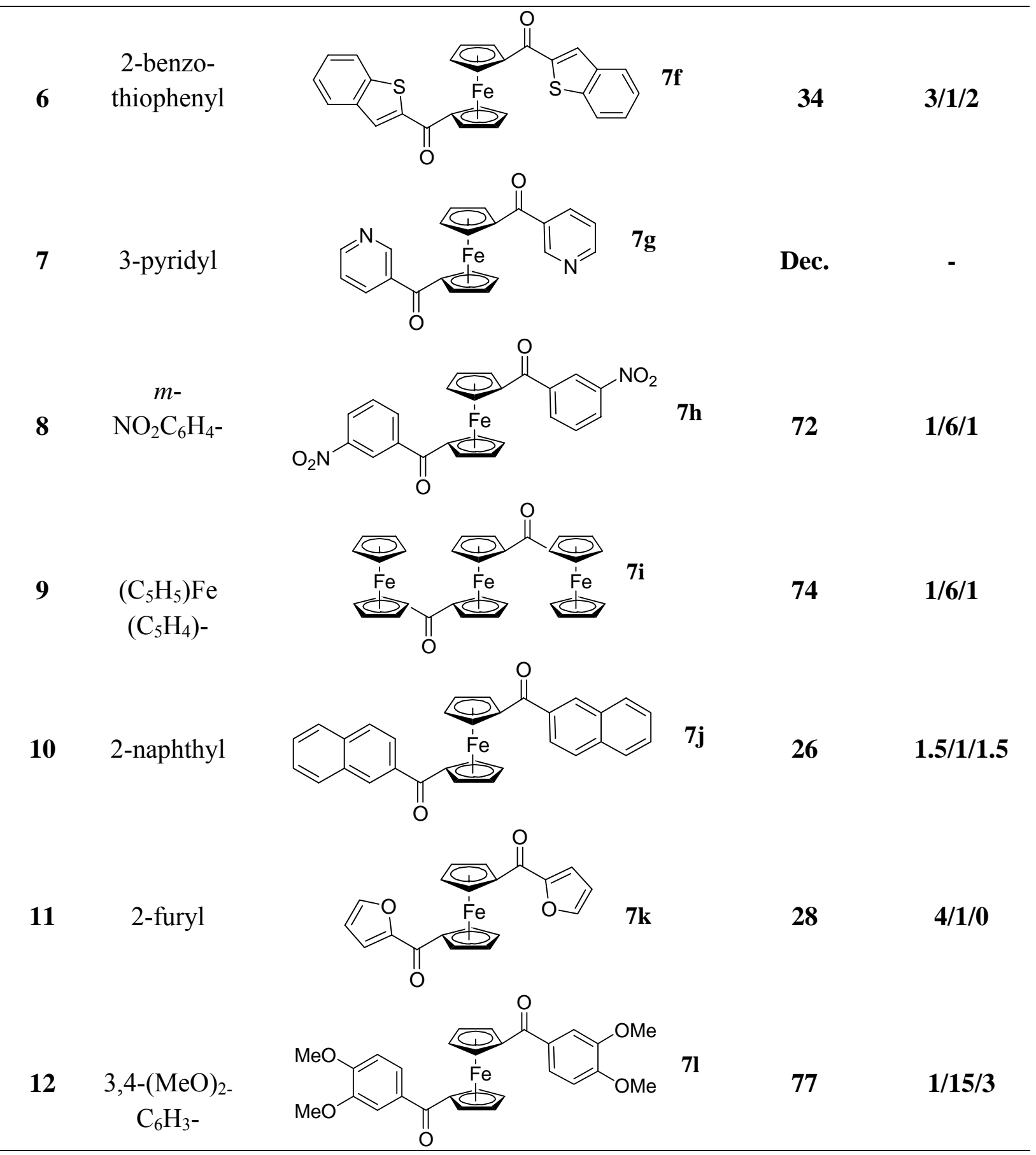

${ }^{a}$ Reaction conditions: thiol ester ( 1 equiv.), $\mathrm{Ar}-\mathrm{B}(\mathrm{OH})_{2}$ (6 equiv.), $\mathrm{Pd}_{2}(\mathrm{dba})_{3} 2.5 \mathrm{~mol} \%$, TFP (tri2-furylphosphine) $7.5 \mathrm{~mol} \%$, CuTC (7 equiv.), THF, $55^{\circ} \mathrm{C}, 18 \mathrm{~h}$.

${ }^{\mathrm{b}}$ Isolated yields. 
Unlike the reaction with mono thiol ester 3, the lack of reactivity of dithiol ester 5 was not a problem since just a small amount of it was recovered at the end of the reactions. The yields of the $1,1^{\prime}$-ferrocenyl diketones were from modest to good.

This time, however, variable quantities of the mono cross-coupled product 8 were isolated in most cases. Attempts were made to obtain predominantly the monocoupled product $\mathbf{8}$ so as to be able to prepare non-symmetrical diketones $\mathrm{Ar}_{1} \mathrm{COFcCOAr} 2$. However, even using lesser amounts of boronic acids, the symmetrical diketones $\operatorname{Ar}_{1} \mathrm{COFcCOAr}_{1} 7$, started to form even at the early stages of the reaction. The double cross-coupling reaction worked efficiently for substituted phenylboronic acids with both electron-releasing and electron-withdrawing groups (entries 1-5, 8,12 ). Of this series, the $m$-nitro derivative was present at the end of the reaction along with the highest amounts of unreacted 5 and monocoupled derivative 8 (entry 8). This result was in sharp contrast with the other example of a reaction with an electron-withdrawing substituted boronic acid, i.e., p-acyl group (entry 5), where only traces of 5 were observed along with the desired product. Some features of the double cross-couplings paralleled those observed in the monocouplings described above. For example, naphthyl derivative (entry 10) was obtained in low yield and appeared as the minor product of the reaction mixture. Similarly, heteroaryl analogues were formed only in poor yield (entries 6 and 11), except for the pyridyl case, which gave decomposition (entry 7). Finally, 1,1'-bisferrocenoylferrocene was obtained in very good yield under the reaction conditions presented in this work (entry 9).

In conclusion, the Liebeskind-Srögl cross-coupling reaction was very efficient to prepare a family of ferrocene-contaning ketones. This process displayed excellent tolerance to different functional groups, with the exception of heterocyclic analogues where the products were obtained either in poor yields or not at all. This methodology represents a more convenient and versatile alternative to the known Friedel-Crafts protocol. The reactivity of thiol esters $\mathbf{3}$ and $\mathbf{5}$ is being studied in the Fukuyama reaction ${ }^{16}$ as well as the reactivity and uses of the ketones prepared. The results will be presented in due course.

\section{Experimental Section}

General Procedures. ${ }^{1} \mathrm{H}$ and ${ }^{13} \mathrm{C}$ NMR spectra were recorded on a Varian Gemini 200 $(200 \mathrm{MHz})$ spectrometer in deuteriochloroform $\left(\mathrm{CDCl}_{3}\right)$ with either tetramethylsilane (TMS) $(0.0 \mathrm{ppm})$ or chloroform $\left(7.26 \mathrm{ppm}{ }^{1} \mathrm{H}, 77.23 \mathrm{ppm}{ }^{13} \mathrm{C}\right)$ as internal reference unless otherwise indicated. Data are reported in the following order: chemical shift in ppm $(\delta)$, multiplicities (br (broadened), s (singlet), d (doublet), t (triplet), q (quartet), sex (sextet), hep (heptet), $\mathrm{m}$ (multiplet), exch (exchangeable), app (apparent)), coupling constants, $\mathrm{J}(\mathrm{Hz})$, and integration. Infrared spectra were recorded on a Perkin Elmer FTIR 1600 series spectrophotometer. Peaks are reported $\left(\mathrm{cm}^{-1}\right)$ with the following relative intensities: s (strong, 67-100\%), $\mathrm{m}$ (medium, 4067\%), and w (weak 20-40\%). Analytical thin-layer chromatography was performed on Merck silica gel plates with F-254 indicator. Visualization was accomplished by UV light, iodine, 
phosphomolybdic acid or $p$-anisaldehyde solutions. All reactions were performed under dry $\mathrm{N}_{2}$ atmosphere in oven- and flame-dried glassware.

Starting Materials. THF was distilled from sodium and benzophenone and stored under $\mathrm{N}_{2}$. Ferrocene, oxalyl chloride, $\mathrm{Pd}_{2} \mathrm{dba}_{3}$, trifurylphosphine, 4-methylbenzenthiol and boronic acids were commercially available and used as received. DMF was deoxygenated and dried over molecular sieves under $\mathrm{N}_{2}$, triethylamine was distilled from $\mathrm{CaH}_{2}$ under $\mathrm{N}_{2}$. CuTC was prepared as reported in the literature. ${ }^{17}$

Ferrocenylthiolester (3). Ferrocenylcarboxylic acid (150 $\mathrm{mg}, 0.65 \mathrm{mmol}$ ) was placed into a round-bottom flask equipped with a stir-bar under $\mathrm{N}_{2}$. Chloroform (10 mL, dry deoxygenated) and DMF (60 mg, $0.82 \mathrm{mmol}$ ) were added and the reaction mixture was placed into an ice-bath. Oxalyl chloride $(99.0 \mathrm{mg}, 0.78 \mathrm{mmol})$ was added dropwise at that temperature via syringe. The mixture was stirred for $10 \mathrm{~min}$. at $0{ }^{\circ} \mathrm{C}$, and then at $25{ }^{\circ} \mathrm{C}$ for $12 \mathrm{~h}$. The solvent was then evaporated in vacuo and the mixture dissolved in benzene. $p$-Methylbenzene thiol $(97 \mathrm{mg}$, $0.78 \mathrm{mmol}$ ) was added followed by the addition of a benzene $(1.0 \mathrm{~mL})$ solution of triethylamine (79 mg, $0.78 \mathrm{mmol}$ ). The solution was stirred for $14 \mathrm{~h}$ at $25^{\circ} \mathrm{C}$, the solvent was then removed in vacuo, ether $(10 \mathrm{~mL})$ was added and the crude material was washed with water $(3 \times 20 \mathrm{~mL})$. The organic extracts were dried over $\mathrm{MgSO}_{4}$, filtered and the solvent removed in vacuo. The product $(180.0 \mathrm{mg}, 82 \%)$ was obtained as orange-red crystals. TLC (silica gel, 20\% AcOEt/hexanes, $R_{f}=$ 0.59); Flash chromatography on silica gel, AcOEt/hexanes gradient; mp $94-96{ }^{\circ} \mathrm{C}$ (hexanes); IR (film, cm $\left.{ }^{-1}\right) 3100$ (w), 3060 (w), 1654 (s), 801 (s); ${ }^{1} \mathrm{H}$ NMR (200 MHz, $\left.\mathrm{CDCl}_{3}\right) \delta 7.40$ (d, 2H), 7.25 (d, 2H), 4.94 (app. t, $J=1.8 \mathrm{~Hz}, 2 \mathrm{H}), 4.54$ (app. t, $J=2.0 \mathrm{~Hz}, 2 \mathrm{H}), 4.30$ (s, 5H), 2.40 (s, $3 \mathrm{H}) .{ }^{13} \mathrm{C}$ NMR $\left(75.5 \mathrm{MHz}, \mathrm{CDCl}_{3}\right) \delta 192.3,139.6,135.2,130.1,124.4,79.0,72.1,70.9,69.3$, 21.6. Anal. Calcd for $\mathrm{C}_{18} \mathrm{H}_{16} \mathrm{FeOS}$ : C, 64.30; H, 4.80; S, 9.54. Found: C, 64.39; H, 4.89; S, 9.50.

Synthesis of 1,1'-ferrocenedicarboxylic acid (4). Into a dry two-necked $250 \mathrm{~mL}$ flash was placed ferrocene $(5.1 \mathrm{~g}, 27.5 \mathrm{mmol})$, dry hexanes $(45 \mathrm{~mL})$ and $N, N$-tetramethylethylene-diamine (TMEDA) (6.7 g, $57.8 \mathrm{mmol}$ ) under $\mathrm{N}_{2}$. Once ferrocene dissolved, $n$-BuLi (57.8 mmol, 1.8M hexanes) was added at $25^{\circ} \mathrm{C}$. The reaction mixture was stirred for $12 \mathrm{~h}$ and the stirring stopped. The supernatant was removed via cannula and the remaining solid washed with fresh dry hexanes $(2 \times 25 \mathrm{~mL})$. An excess amount of dry ice was then directly added to the reaction mixture under a positive $\mathrm{N}_{2}$ pressure. ${ }^{18}$ The mixture was then stirred for $12 \mathrm{~h}$ and then, a saturated aq. $\mathrm{NH}_{4} \mathrm{Cl}$ solution was added $(15 \mathrm{~mL})$ followed by $1 \mathrm{~N} \mathrm{HCl}(15 \mathrm{~mL})$. The precipitate was filtered, washed with ether $(3 \times 15 \mathrm{~mL})$ and vacuum dried. A second crop was obtained by treating the organic extracts with $2.5 \mathrm{M} \mathrm{NaOH}$ solution until $\mathrm{pH} 13$. The aqueous phase was washed with ether $(3 \times 15 \mathrm{~mL})$, and acidified with $1 \mathrm{~N} \mathrm{HCl}$ until no more acid precipitated. The solid was filtered and washed with ether $(3 \times 15 \mathrm{~mL})$ and vacuum dried. A total amount of broth crops gave $5.91 \mathrm{~g}(79 \%)$ of the acid as an orange powder. ${ }^{1} \mathrm{H}$ NMR (DMSO- $\left.d_{6}\right) \delta 4.69$ (app t, $J=$ $2.2 \mathrm{~Hz}, 4 \mathrm{H}), 4.45$ (app t, $J=1.8 \mathrm{~Hz}, 4 \mathrm{H})\left(\right.$ lit $\left.^{19}\right)$.

Ferrocenylbisthiolester (5). 1,1'-Ferrocenyldicarboxylic acid (300 mg, $1.1 \mathrm{mmol}$ ) was placed into a round-bottom flask equipped with a stir-bar under $\mathrm{N}_{2}$. Chloroform $(10 \mathrm{~mL}$, dry 
deoxygenated) and DMF (60 $\mathrm{mg}, 0.82 \mathrm{mmol}$ ) were added and the reaction mixture was placed into an ice-bath. Oxalyl chloride $(99.0 \mathrm{mg}, 0.78 \mathrm{mmol}$ ) was added dropwise at that temperature via syringe. The mixture was stirred for $10 \mathrm{~min}$. at $0{ }^{\circ} \mathrm{C}$, and then at $25{ }^{\circ} \mathrm{C}$ for $12 \mathrm{~h}$. The solvent was then evaporated in vacuo and the mixture dissolved in benzene. $p$-Methylbenzene thiol $(97 \mathrm{mg}, 0.78 \mathrm{mmol})$ was added followed by the addition of a benzene $(1.0 \mathrm{~mL})$ solution of triethylamine $\left(79 \mathrm{mg}, 0.78 \mathrm{mmol}\right.$ ). The solution was stirred for $14 \mathrm{~h}$ at $25^{\circ} \mathrm{C}$, the solvent was then removed in vacuo, ether $(10 \mathrm{~mL})$ was added and the crude material was washed with water ( 3 x $20 \mathrm{~mL}$ ). The organic extracts were dried over MgSO4, filtered and the solvent removed in vacuo. The product $(180.0 \mathrm{mg}, 82 \%)$ was obtained as orange-red crystals. Chromatographic purification by Flash chromatography on silica gel using an EtOAc/hexanes gradient. TLC (20\% AcOEt/hexanes, $R_{f}=0.41$ ); mp 153-155 ${ }^{\circ} \mathrm{C}$ (hexanes); IR (film, $\mathrm{cm}^{-1}$ ) $3080(\mathrm{w}), 2921(\mathrm{w}), 1673$ (s), 806 (s). ${ }^{1} \mathrm{H}$ NMR (200 MHz, $\left.\mathrm{CDCl}_{3}\right) \delta 7.40$ (d, 4H), 7.25 (d, 4H), 5.01 (app. t, $\left.J=2.0,4 \mathrm{H}\right)$, 4.60 (app. t, $J=2.0,4 \mathrm{H}), 2.41(\mathrm{~s}, 6 \mathrm{H}) .{ }^{13} \mathrm{C} \mathrm{NMR}\left(75.5 \mathrm{MHz}, \mathrm{CDCl}_{3}\right) \delta 191.5,139.8,135.2$, 130.2, 123.9, 80.8, 74.6, 71.2, 21.6. Anal. Calcd for $\mathrm{C}_{26} \mathrm{H}_{22} \mathrm{FeO}_{2} \mathrm{~S}_{2}$ : C, 64.20; H, 4.56; S, 13.18. Found: C, 64.64; H, 4.65; S, 13.09 .

\section{General procedure for the synthesis of ferrocenyl monoketones}

A Schlenk tube was charged with thiol ester 3 (1.0 equiv.), CuTC (4 equiv), boronic acid (3 equiv.), $\mathrm{Pd}_{2} \mathrm{dba}_{3}(2.5 \mathrm{~mol} \%)$ and TFP $(7.5 \mathrm{~mol} \%)$ and THF $(5 \mathrm{~mL})$ under $\mathrm{N}_{2}$. The reaction mixture was stirred at $55-60{ }^{\circ} \mathrm{C}$ for $18 \mathrm{~h}$. At the end of this period, the mixture was allowed to reach $25{ }^{\circ} \mathrm{C}$. Diethyl ether $(5 \mathrm{~mL})$ was added and the mixture was washed with a $1 \%$ aq. $\mathrm{NaOH}$ solution ( $3 \times 10 \mathrm{~mL}$ ). The organic layer was dried over MgSO4, filtered and the solvent removed in vacuo. The products were purified by Flash chromatography on silica gel using a EtOAc/hexanes gradient.

\section{General procedure for the synthesis of $1,1^{\prime}$-ferrocenyldiketones}

A Schlenk tube was charged with thiol ester 5 (1.0 equiv.), CuTC (7 equiv), boronic acid (6 equiv.), $\mathrm{Pd}_{2} \mathrm{dba}_{3}(2.5 \mathrm{~mol} \%)$ and TFP $(7.5 \mathrm{~mol} \%)$ and THF $(5 \mathrm{~mL})$ under $\mathrm{N}_{2}$. The reaction mixture was stirred at $55-60{ }^{\circ} \mathrm{C}$ for $18 \mathrm{~h}$. At the end of this period, the mixture was allowed to reach $25{ }^{\circ} \mathrm{C}$. Diethyl ether $(5 \mathrm{~mL})$ was added and the mixture was washed with a $1 \%$ aq. $\mathrm{NaOH}$ solution $(3 \times 10 \mathrm{~mL})$. The organic layer was dried over $\mathrm{MgSO} 4$, filtered and the solvent removed in vacuo. The products were purified by Flash chromatography on silica gel using a EtOAc/hexanes gradient.

Ketone 6a. Red crystals (92\%). TLC (20\% AcOEt/hexanes, $\left.\mathrm{R}_{\mathrm{f}}=0.55\right)$; mp 106-107 ${ }^{\circ} \mathrm{C}$. ${ }^{1} \mathrm{H}$ NMR $\left(200 \mathrm{MHz}, \mathrm{CDCl}_{3}\right) \delta 7.91(\mathrm{dd}, \mathrm{J}=1.4,8.0 \mathrm{~Hz}, 2 \mathrm{H}), 7.51(\mathrm{~m}, 3 \mathrm{H}), 4.92($ app. t, J = $1.8 \mathrm{~Hz}$, 2H), 4.60 (app. t, J = 2.2 Hz, 2H), $4.22(\mathrm{~s}, 5 \mathrm{H}) .{ }^{13} \mathrm{C} \mathrm{NMR}\left(75.5 \mathrm{MHz}, \mathrm{CDCl}_{3}\right) \delta 199.3,140.0$, $131.7,128.4,128.2,78.3,72.7,71.7,70.4\left(\right.$ lit $\left.^{20,21}\right)$.

Ketone 6e. Orange solid (87\%). TLC (20\% AcOEt/hexanes, $\left.R_{f}=0.38\right)$; mp $94-95{ }^{\circ} \mathrm{C}$ (hexanes); IR (film, cm ${ }^{-1}$ ) 3089 (w), 2921 (w), 1632 (s), 1599 (s), 840 (m), 769 (m); ${ }^{1} \mathrm{H}$ NMR (200 MHz, $\left.\mathrm{CDCl}_{3}\right) \delta 7.97(\mathrm{~d}, 2 \mathrm{H}), 7.43(\mathrm{~m}, 5 \mathrm{H}), 7.05(\mathrm{~d}, 2 \mathrm{H}), 5.16(\mathrm{~s}, 2 \mathrm{H}), 4.92$ (app. t, $\left.J=1.8 \mathrm{~Hz}, 2 \mathrm{H}\right)$, 
4.22 (s, 5H), 4.57 (app. t, $J=1.8 \mathrm{~Hz}, 2 \mathrm{H}) .{ }^{13} \mathrm{C} \mathrm{NMR}\left(75.5 \mathrm{MHz}, \mathrm{CDCl}_{3}\right) \delta$ 197.6, 161.8, 136.6, 132.8, 130.6, 128.9, 128.4, 127.7, 114.5, 78.9, 72.4, 71.7, 70.4, 70.3. Anal. Calcd for $\mathrm{C}_{24} \mathrm{H}_{20} \mathrm{FeO}_{2}$ : C, 72.74; H, 5.09. Found: C, 72.56; H, 5.44.

Ketone 6f. Dark red crystals (78\%). TLC (20\% AcOEt/hexanes, $\left.R_{f}=0.29\right) ; \mathrm{mp} \mathrm{103-104}{ }^{\circ} \mathrm{C}$ (hexanes); IR (film, cm ${ }^{-1}$ ) 3054 (w), 2916 (w), 1681 (s), 1638 (s), 818 (m); ${ }^{1} \mathrm{H} \mathrm{NMR} \mathrm{(200} \mathrm{MHz,}$ $\left.\mathrm{CDCl}_{3}\right) \delta 8.07$ (d, 2H), $7.96(\mathrm{~d}, 2 \mathrm{H}), 4.89$ (app. t, $\left.J=2.2 \mathrm{~Hz}, 2 \mathrm{H}\right), 4.64$ (app. t, $J=2.0 \mathrm{~Hz}, 2 \mathrm{H}$ ), $4.24(\mathrm{~s}, 5 \mathrm{H}), 2.70(\mathrm{~s}, 3 \mathrm{H}) .{ }^{13} \mathrm{C}$ NMR $\left(75.5 \mathrm{MHz}, \mathrm{CDCl}_{3}\right) \delta$ 198.7, 197.8, 143.8. 139.2, 128.4, 128.3, 77.9, 73.2, 71.6, 70.5, 27.0. Anal. Calcd for $\mathrm{C}_{19} \mathrm{H}_{16} \mathrm{FeO}_{2}$ : C, 68.70; H, 4.85. Found: C, 68.84; H, 4.65.

Ketone 6g. Purple red crystals (77\%). TLC (20\% AcOEt/hexanes, $\left.R_{f}=0.56\right) ; \mathrm{mp} 157-159{ }^{\circ} \mathrm{C}$ (hexanes); IR (film, cm ${ }^{-1}$ ) 3100 (w), 2919 (w), 1613 (s), 1513 (s), 1446 (s), 824 (m), 759 (m); ${ }^{1} \mathrm{H}$ NMR $\left(200 \mathrm{MHz}, \mathrm{CDCl}_{3}\right) \delta 8.15(\mathrm{~s}, 1 \mathrm{H}), 7.95(\mathrm{~m}, 2 \mathrm{H}), 7.46(\mathrm{~m}, 2 \mathrm{H}), 5.12$ (app. t, $J=1.8 \mathrm{~Hz}$, 2H), 4.66 (app. t, $J=1.8 \mathrm{~Hz}, 2 \mathrm{H}), 2.28(\mathrm{~s}, 5 \mathrm{H}) .{ }^{13} \mathrm{C} \mathrm{NMR}\left(75.5 \mathrm{MHz}, \mathrm{CDCl}_{3}\right) \delta 190.9,143.9$, $141.8,139.2,128.5,127.1,125.8,125.1,123.0,78.9,72.9,71.3,70.8$. Anal. Calcd for $\mathrm{C}_{19} \mathrm{H}_{14} \mathrm{FeOS}$ : C, 65.91; H, 4.08; S, 9.26. Found: C, 66.12; H, 4.01; S, 9.33.

Ketone 6d. Brown-red crystals (98\%). TLC (20\% AcOEt/hexanes, $\left.R_{f}=0.43\right) ; \mathrm{mp} \mathrm{79-80}{ }^{\circ} \mathrm{C}$ (hexanes); IR (film, cm ${ }^{-1}$ ) 3095 (w), 2931 (w), 1632 (s), 1599 (s), 1377 (s), 843 (m); ${ }^{1} \mathrm{H}$ NMR $\left(200 \mathrm{MHz}, \mathrm{CDCl}_{3}\right) \delta 7.97$ (d, 2H), 6.97 (d, 2H), 4.92 (bs, 2H), 4.57 (bs, 2H), 4.24 (s, 5H), 3.90 (s, 3H). ${ }^{13} \mathrm{C} \mathrm{NMR}\left(75.5 \mathrm{MHz}, \mathrm{CDCl}_{3}\right) \delta$ 197.7. 162.6, 132.5, 130.6, 113.6, 78.9, 72.3, 71.7, 70.4, 55.6 (lit. $^{22}$ ).

Ketone 6c. Red oil (79\%). TLC (20\% AcOEt/hexanes, $\left.R_{f}=0.62\right)$; IR (film, cm $\left.{ }^{-1}\right) 3055(\mathrm{w}), 2918$ (w), 1638 (s), 824 (m); ${ }^{1} \mathrm{H}$ NMR $\left(200 \mathrm{MHz}, \mathrm{CDCl}_{3}\right.$ ) $\delta 7.71$ (bs, 2H), 7.36 (m, 2H), 4.92 (app. t, $J$ $=1.8 \mathrm{~Hz}, 2 \mathrm{H}), 4.59$ (app. $\mathrm{t}, J=1.8 \mathrm{~Hz}, 2 \mathrm{H}), 4.22(\mathrm{~s}, 5 \mathrm{H}), 2.46(\mathrm{~s}, 3 \mathrm{H}) .{ }^{13} \mathrm{C} \mathrm{NMR}(75.5 \mathrm{MHz}$, $\left.\mathrm{CDCl}_{3}\right) \delta 199.6,140.0,138.2,132.4,128.8,128.2,125.5,78.5,72.7,71.8,70.4,21.6\left(1 \mathrm{it}^{21}\right)$.

Ketone 6i. Orange solid (50\%). TLC (20\% AcOEt/hexanes, $\left.\mathrm{R}_{\mathrm{f}}=0.51\right) ; \mathrm{mp} 141-142{ }^{\circ} \mathrm{C}$ (hexanes); IR (film, cm ${ }^{-1}$ ) 3085 (w), 2924 (m), 1643 (s), 1531 (s), 1377 (m), 1349 (m), 825 (m); ${ }^{1} \mathrm{H}$ NMR $\left(200 \mathrm{MHz}, \mathrm{CDCl}_{3}\right) \delta 9.00$ (app. t, J = 1.8 Hz, 1H), $8.20(\mathrm{~m}, 1 \mathrm{H}), 8.42(\mathrm{~m}, 1 \mathrm{H}), 7.70(\mathrm{t}$, $\mathrm{J}=7.8 \mathrm{~Hz}, 1 \mathrm{H}$ ), 4.91 (app. $\mathrm{t} \mathrm{J}=1.8 \mathrm{~Hz}, 2 \mathrm{H}$ ), 4.71 (app. $\mathrm{t}, \mathrm{J}=1.8 \mathrm{~Hz}, 2 \mathrm{H}), 4.31$ (s, 5H). ${ }^{13} \mathrm{C}$ NMR $\left(75.5 \mathrm{MHz}, \mathrm{CDCl}_{3}\right) \delta 196.9,148.0,141.0,134.2,129.9,126.2,123.6,77.4,73.5,71.6$, 70.7. Anal. Calcd for $\mathrm{C}_{17} \mathrm{H}_{13} \mathrm{FeNO}_{3}$ : C, 60.93; H, 3.91; N, 4.18. Found: C, 60.86; H, 4.29; N, 4.01.

Ketone 6j. Brown-orange solid (69\%). TLC (20\% AcOEt/hexanes, $\left.R_{f}=0.52\right) ;{ }^{1} \mathrm{H}$ NMR (200 $\mathrm{MHz} \mathrm{CDCl}_{3}$ ) $\delta 5.00$ (app. t, $J=1.8 \mathrm{~Hz}, 4 \mathrm{H}$ ). 4.54 (app. t, $J=2.0 \mathrm{~Hz}, 4 \mathrm{H}$ ), $4.21(\mathrm{~s}, 10 \mathrm{H}) .{ }^{13} \mathrm{C}$ $\operatorname{NMR}\left(75.5 \mathrm{MHz}, \mathrm{CDCl}_{3}\right) \delta 199.6,80.6,71.7,70.8,70.2$ (lit. $\left.^{23}\right)$.

Ketone 6l. Dark red oil (22\%). TLC (20\% AcOEt/hexanes, $\left.R_{f}=0.52\right)$; IR (film, cm $\left.{ }^{-1}\right) 3054(\mathrm{w})$, 2924 (m), 1622 (s), 1470 (s), 817 (m); ${ }^{1} \mathrm{H}$ NMR (200 MHz, CDCl $) \delta 7.65$ (m, 1H), 7.33 (d, J= $3.2 \mathrm{~Hz}, 1 \mathrm{H}$ ), 6.58 (dd, $J=4.0,1.8 \mathrm{~Hz}, 1 \mathrm{H}$ ), 5.18 (app. t, $J=1.8 \mathrm{~Hz}, 2 \mathrm{H}$ ), 4.61 (app. t, $J=2.0 \mathrm{~Hz}$, 2H), $4.20(\mathrm{~s}, 5 \mathrm{H}) .{ }^{13} \mathrm{C} \mathrm{NMR}\left(75.5 \mathrm{MHz}, \mathrm{CDCl}_{3}\right) \delta 185.0,153.9,145.4,116.9,112.2,72.7,71.0$, 70.4. HRMS (EI) Calcd for $\mathrm{C}_{15} \mathrm{H}_{12} \mathrm{FeO}_{2}$ 280.0187. Found 280.0185. 
Ketone 6k. Red oil (57\%). TLC (20\% AcOEt/hexanes, $\left.R_{f}=0.47\right)$; IR (film, cm $\left.{ }^{-1}\right) 3054(\mathrm{w})$, 2849 (w), 1637 (m), 884 (m), 738 (s); ${ }^{1} \mathrm{H}$ NMR (200 MHz, CDCl 3 ) $\delta 8.46$ (bs, 1H), 7.97 (m, 4H), 7.59 (m, 2H), 4.99 (app. t, $J=1.8 \mathrm{~Hz}, 2 \mathrm{H}$ ), 4.63 (app. t, $J=1.8 \mathrm{~Hz}, 2 \mathrm{H}$ ), 4.24 (s, 5H). ${ }^{13} \mathrm{C}$ NMR (75.5 MHz, $\left.\mathrm{CDCl}_{3}\right) \delta 199.2,137.2,135.0,132.6,129.3,129.0,128.3,128.0,127.9,126.9$, 125.1, 78.6, 72.8, 71.8, 70.5. HRMS (EI) Calcd for $\mathrm{C}_{21} \mathrm{H}_{16} \mathrm{FeO} 340.0550$. Found 340.0553.

Ketone 6b. Red-orange solid (99\%). TLC (20\% AcOEt/hexanes, $\left.R_{f}=0.66\right)$; IR (film, cm $\left.{ }^{-1}\right) 2919$ (w), 2856 (w), 1634 (s), 1445 (m), 824 (m); ${ }^{1} \mathrm{H}$ NMR (200 MHz, CDCl ${ }_{3}$ ) $\delta 7.83$ (d, 2H), 7.28 (d, 2H), 4.91 (app. t, $J=2.0 \mathrm{~Hz}, 2 \mathrm{H}), 4.58$ (app. t, $J=1.8 \mathrm{~Hz}, 2 \mathrm{H}), 4.21(\mathrm{~s}, 5 \mathrm{H}), 2.44(\mathrm{~s}, 3 \mathrm{H}) .{ }^{13} \mathrm{C}$ NMR (75.5 MHz, $\left.\mathrm{CDCl}_{3}\right) \delta 198.9,142.3,137.3,129.1,128.5,78.7,72.6,71.7,70.4,21.8$ (lit. $^{21}$ ). Dione 7a. Dark red crystals (61\%). TLC (20\% AcOEt/hexanes, $\left.R_{f}=0.25\right) ; \mathrm{mp} \mathrm{102-103}{ }^{\circ} \mathrm{C}$ (hexanes); IR (film, cm ${ }^{-1}$ ) 3061 (w), 2916 (w), 1640 (s), 856 (s), 729 (s), 698 (s). ${ }^{1} \mathrm{H}$ NMR (200 $\left.\mathrm{MHz} \mathrm{CDCl}_{3}\right) \delta 7.79(\mathrm{dd}, J=1.6,8.0 \mathrm{~Hz}, 4 \mathrm{H}), 7.47(\mathrm{~m}, 6 \mathrm{H}), 4.93$ (app. t, $\left.J=1.8 \mathrm{~Hz}, 4 \mathrm{H}\right), 4.59$ (app. t, $J=2.2 \mathrm{~Hz}, 4 \mathrm{H}) .{ }^{13} \mathrm{C} \mathrm{NMR}\left(75.5 \mathrm{MHz}, \mathrm{CDCl}_{3}\right) \delta 198.2,139.3,132.1,128.5,128.3,79.7$, 74.8, 73.3 (lit. $^{20,21}$ ).

Dione 7d. Orange solid (63\%). TLC (30\% AcOEt/hexanes, $\left.R_{f}=0.43\right) ; \mathrm{mp} \mathrm{191-192}{ }^{\circ} \mathrm{C}$ (hexanes); IR (film, cm ${ }^{-1}$ ) 3088 (w), 2922 (w), 1631 (s), 1601 (s), 843 (m), 770 (m); ${ }^{1} \mathrm{H}$ NMR $\left(200 \mathrm{MHz}, \mathrm{CDCl}_{3}\right) \delta 7.84(\mathrm{~d}, 4 \mathrm{H}), 7.41(\mathrm{~m}, 10 \mathrm{H}), 7.00(\mathrm{~d}, 4 \mathrm{H}), 5.18$ (app. t, $\left.J=2.0 \mathrm{~Hz}, 4 \mathrm{H}\right)$, 5.14 (s, 4H), 4.56 (app. t, $J=2.0 \mathrm{~Hz}, 4 \mathrm{H}) .{ }^{13} \mathrm{C} \mathrm{NMR}\left(75.5 \mathrm{MHz}, \mathrm{CDCl}_{3}\right) \delta 196.5,162.1,136.5$, 132.2, 130.8, 129.0, 128.4, 127.7, 114.6, 80.3, 74.4, 73.4, 70.3. Anal. Calcd for $\mathrm{C}_{38} \mathrm{H}_{30} \mathrm{FeO}_{4}$ : C, 75.25; H, 4.99. Found: C, 75.14; H, 5.21.

Dione 7e. Dark red crystals (59\%). TLC (30\% AcOEt/hexanes, $\left.R_{f}=0.16\right) ; \mathrm{mp} 210-211{ }^{\circ} \mathrm{C}$ (hexanes); IR (film, cm ${ }^{-1}$ ) 3100 (w), 2922 (w), 1683 (s), 1639 (s), 845 (m); ${ }^{1} \mathrm{H} \mathrm{NMR} \mathrm{(200} \mathrm{MHz,}$ $\left.\mathrm{CDCl}_{3}\right) \delta 8.00(\mathrm{~d}, 4 \mathrm{H}), 7.83(\mathrm{~d}, 4 \mathrm{H}), 4.90$ (app. t, $\left.J=2.0 \mathrm{~Hz}, 4 \mathrm{H}\right), 4.64$ (app. t, $J=2.0 \mathrm{~Hz}, 4 \mathrm{H}$ ), $2.68(\mathrm{~s}, 6 \mathrm{H}) .{ }^{13} \mathrm{C} \mathrm{NMR}\left(75.5 \mathrm{MHz}, \mathrm{CDCl}_{3}\right) \delta 197.7,197.3,142.8,139.5,128.5,128.4,79.4$, 75.0, 73.4, 27.1. Anal. Calcd for $\mathrm{C}_{28} \mathrm{H}_{22} \mathrm{FeO}_{4}$ : C, 70.31; H, 4.64. Found: C, 70.53; H, 4.88.

Dione 7f. Dark red oil (34\%). TLC (20\% AcOEt/hexanes, $\left.R_{f}=0.27\right)$. IR (film, $\left.\mathrm{cm}^{-1}\right) 3080(\mathrm{w})$, 2924 (w), 1614 (m), 1514 (m), 1446 (m), 834 (m), 760 (m); ${ }^{1} \mathrm{H}$ NMR (200 MHz, CDCl $) \delta 8.03$ (s, 2H), $7.82(\mathrm{~m}, 4 \mathrm{H}), 7.41(\mathrm{~m}, 4 \mathrm{H}), 5.16$ (app. t, $J=2.0 \mathrm{~Hz}, 4 \mathrm{H}), 4.70$ (app. t, $J=1.8 \mathrm{~Hz}, 4 \mathrm{H})$. ${ }^{13} \mathrm{C}$ NMR $\left(75.5 \mathrm{MHz}, \mathrm{CDCl}_{3}\right) \delta 189.8,143.4,142.0,139.2,129.1,127.3,126.0,125.2,122.9$, 80.4, 75.0, 73.1. HRMS (EI) Calcd for $\mathrm{C}_{28} \mathrm{H}_{18} \mathrm{FeO}_{2} \mathrm{~S}_{2}$ 506.0098. Found 506.0096.

Dione 7c. Dark red solid (68\%). TLC (20\% AcOEt/hexanes, $\left.R_{f}=0.09\right) ; \mathrm{mp} 142-143{ }^{\circ} \mathrm{C}$ (hexanes); IR (film, cm ${ }^{-1}$ ) 3040 (w), 2917 (w), 2850 (w), 1599 (m), 843 (m); ${ }^{1} \mathrm{H}$ NMR (200 $\left.\mathrm{MHz} \mathrm{CDCl}_{3}\right) \delta 7.84$ (d, 4H), 6.90 (d, 4H), 4.91 (app. t, $\left.J=1.4 \mathrm{~Hz}, 4 \mathrm{H}\right), 4.56$ (bs, 4H), 3.90 (s, $6 \mathrm{H}) .{ }^{13} \mathrm{C}$ NMR $\left(75.5 \mathrm{MHz}, \mathrm{CDCl}_{3}\right) \delta 196.5,162.9,132.0,130.7,113.7,80.3,74.4,73.3,55.7$. Anal. Calcd for $\mathrm{C}_{26} \mathrm{H}_{22} \mathrm{FeO}_{4}$ : C, 68.74; H, 4.88. Found: C, 68.89; H, 4.95.

Dione 7b. Bright red crystals (77\%). TLC (20\% AcOEt/hexanes, $\left.R_{f}=0.30\right) ; \mathrm{mp} 86-88{ }^{\circ} \mathrm{C}$ (hexanes); IR (film, cm ${ }^{-1}$ ) 2922 (m), 1712 (w), 1642 (s), 813 (m); ${ }^{1} \mathrm{H} \mathrm{NMR} \mathrm{(200} \mathrm{MHz,} \mathrm{CDCl} 3$ ) $\delta$ 7.57 (m, 4H), 7.33 (m, 4H), 4.92 (app. t, $J=2.2 \mathrm{~Hz}, 4 \mathrm{H}$ ), 4.58 (app. t, $J=2.2 \mathrm{~Hz}, 4 \mathrm{H}$ ), 2.41 (s, $6 \mathrm{H}) .{ }^{13} \mathrm{C} \mathrm{NMR}\left(75.5 \mathrm{MHz}, \mathrm{CDCl}_{3}\right) \delta 198.4,139.3,138.3,132.9,128.8,128.3,125.5,79.7,74.8$, 73.3, 21.6. Anal. Calcd for $\mathrm{C}_{26} \mathrm{H}_{22} \mathrm{FeO}_{2}$ : C, 73.95; H, 5.25. Found: C, 73.62; H, 5.53. 
Dione 7h. Dark red solid (72\%). TLC (20\% AcOEt/hexanes, $\left.R_{f}=0.13\right) ; \mathrm{mp} 198-199{ }^{\circ} \mathrm{C}$ (hexanes); IR (film, cm ${ }^{-1}$ ) 3089 (w), 2920 (w), 1637 (s), 1531 (s), 1350 (s), 896 (w); ${ }^{1} \mathrm{H}$ NMR $\left(200 \mathrm{MHz}, \mathrm{CDCl}_{3}\right) \delta 8.76(\mathrm{~s}, 2 \mathrm{H}), 8.41(\mathrm{~d}, J=7.0 \mathrm{~Hz}, 2 \mathrm{H}), 8.13(\mathrm{~d}, J=7.2 \mathrm{~Hz}, 2 \mathrm{H}), 7.67$ (t, $J=$ $8.0 \mathrm{~Hz}, 2 \mathrm{H}), 4.98$ (bs, 4H), 4.74 (bs, 4H). ${ }^{13} \mathrm{C} \mathrm{NMR}\left(75.5 \mathrm{MHz}, \mathrm{CDCl}_{3}\right) \delta 195.3,148.1,140.1$, 134.1, 130.0, 126.7, 123.4, 79.1, 75.1, 73.5. Anal. Calcd for $\mathrm{C}_{24} \mathrm{H}_{16} \mathrm{FeN}_{2} \mathrm{O}_{6}$ : C, 59.53; H, 3.33; N, 5.79. Found: C, 59.81; H, 3.66; N, 5.88.

Dione 7i. Orange solid (74\%). TLC (20\% AcOEt/hexanes, $\left.R_{f}=0.16\right) ; \mathrm{mp} 185-187{ }^{\circ} \mathrm{C} \mathrm{dec}$. (hexanes); IR (film, cm ${ }^{-1}$ ) 2928 (m), 1704 (m), 1626 (m); ${ }^{1} \mathrm{H}$ NMR (200 MHz, CDCl 3 ) $\delta 5.02$ (bs, 4H), 4.97 (bs, 4H), 4.55 (bs, 4H), 4.51 (bs, 4H), 4.18 (s, 10H). ${ }^{13} \mathrm{C} \mathrm{NMR}\left(75.5 \mathrm{MHz}, \mathrm{CDCl}_{3}\right)$ $\delta$ 198.9, 81.8, 80.1, 74.0, 72.1, 72.0, 70.8, 70.3. Anal. Calcd for $\mathrm{C}_{32} \mathrm{H}_{26} \mathrm{Fe}_{3} \mathrm{O}_{2}$ : C, 63.00; H, 4.30. Found: C, 62.89; H, 4.25.

Dione 7k. Red oil (28\%). TLC (20\% AcOEt/hexanes, $\left.R_{f}=0.09\right)$; IR (film, $\left.\mathrm{cm}^{-1}\right) 3054(\mathrm{w}), 2916$ (m), 2848 (w), 1625 (s) 1470 (m), 818 (w); ${ }^{1} \mathrm{H}$ NMR (200 MHz, CDCl ${ }_{3}$ ) 7.57 (bs, 2H), 7.30 (s, 2H), 6.55 (m, 2H), 5.18 (app. t, $J=1.8 \mathrm{~Hz}, 4 \mathrm{H}$ ), 4.59 (app. t, $J=1.8 \mathrm{~Hz}, 4 \mathrm{H}) .{ }^{13} \mathrm{C}$ NMR $(75.5$ $\left.\mathrm{MHz}, \mathrm{CDCl}_{3}\right) \delta 183.9,153.6,145.9,117.4,112.3,79.3,74.4,72.7$. HRMS (EI) Calcd for $\mathrm{C}_{20} \mathrm{H}_{14} \mathrm{FeO}_{4}$ 374.0241. Found 374.0244.

Dione 7j. Orange-red solid (26\%). TLC (20\% AcOEt/hexanes, $\left.R_{f}=0.21\right) ; \mathrm{mp} 189-190{ }^{\circ} \mathrm{C}$ (hexanes); IR (film, cm ${ }^{-1}$ ) 3055 (w), 2919 (m), 1674 (m), 810 (s), 740 (s); ${ }^{1} \mathrm{H}$ NMR (200 MHz, $\left.\mathrm{CDCl}_{3}\right) \delta 8.30(\mathrm{~s}, 2 \mathrm{H}), 7.85(\mathrm{~m}, 4 \mathrm{H}), 7.76(\mathrm{~m}, 4 \mathrm{H}), 7.57(\mathrm{~m}, 4 \mathrm{H}), 5.01$ (app. t, $\left.J=2.2 \mathrm{~Hz}, 4 \mathrm{H}\right)$, 4.66 (app. t, $J=2.2 \mathrm{~Hz}, 4 \mathrm{H}) .{ }^{13} \mathrm{C}$ NMR $\left(75.5 \mathrm{MHz}, \mathrm{CDCl}_{3}\right) \delta 197.9,136.4,135.2,132.5,129.4$, 129.2, 128.4, 128.1, 128.0, 126.9, 124.7, 80.0, 74.8, 73.6. Anal. Calcd for $\mathrm{C}_{32} \mathrm{H}_{22} \mathrm{FeO}_{2}$ : C, 77.75; H, 4.49. Found: C, 77.44; H, 4.67.

Dione 7l. Red crystals (77\%). TLC (20\% AcOEt/hexanes, $\left.R_{f}=0.12\right) ; \mathrm{mp} 181-182{ }^{\circ} \mathrm{C}$ (hexanes); IR (film, cm ${ }^{-1}$ ) 3055 (w), 2919 (m), 1674 (m), 810 (s), 740 (s); ${ }^{1} \mathrm{H}$ NMR (200 MHz, CDCl $\left.{ }_{3}\right) \delta$ $7.51(\mathrm{~d}, J=8.2 \mathrm{~Hz}, 2 \mathrm{H}), 7.35$ (bs, 2H), 6.81 (d, $J=8.4 \mathrm{~Hz}, 2 \mathrm{H}), 4.92$ (bs, 4H), 4.58 (bs, 4H), 3.96 $(\mathrm{s}, 6 \mathrm{H}), 3.93(\mathrm{~s}, 6 \mathrm{H}) .{ }^{13} \mathrm{C} \mathrm{NMR}\left(75.5 \mathrm{MHz}, \mathrm{CDCl}_{3}\right) \delta 196.2,152.6,149.0,131.9,122.9,111.0$, 109.9, 80.2, 74.2, 73.4, 56.1. Anal. Calcd for $\mathrm{C}_{28} \mathrm{H}_{26} \mathrm{FeO}_{6}$ : C, 65.38; H, 5.10. Found: C, 65.08; H, 5.05 .

Dione 8d. Orange crystals (5\%). TLC (20\% AcOEt/hexanes, $\left.R_{f}=0.25\right) ; \mathrm{mp} 126-127{ }^{\circ} \mathrm{C}$ (hexanes); IR (film, cm ${ }^{-1}$ ) 2922 (w), 1674 (m), 1634 (m), 809 (m), 770 (m), 697 (m); ${ }^{1} \mathrm{H}$ NMR $\left(200 \mathrm{MHz}, \mathrm{CDCl}_{3}\right) \delta 7.93(\mathrm{~d}, 2 \mathrm{H}), 7.43(\mathrm{~m}, 5 \mathrm{H}), 7.24$ (bs, 4H), $7.04(\mathrm{~d}, 2 \mathrm{H}), 5.14(\mathrm{~s}, 2 \mathrm{H}), 4.99$ (app. t, $J=2.2 \mathrm{~Hz}, 2 \mathrm{H}$ ), 4.91 (app. t, $J=2.0 \mathrm{~Hz}, 2 \mathrm{H}$ ), 4.63 (app. t, $J=2.0 \mathrm{~Hz}, 2 \mathrm{H}$ ), 4.53 (app. t, $J$ $=2.0 \mathrm{~Hz}, 2 \mathrm{H}), 2.38(\mathrm{~s}, 3 \mathrm{H}) .{ }^{13} \mathrm{C} \mathrm{NMR}\left(75.5 \mathrm{MHz}, \mathrm{CDCl}_{3}\right) \delta 196.4,191.7,162.2,139.8,136.5$, $135.1,132.3,130.9,130.1,128.9,128.4,127.7,124.0,114.6,80.7,80.4,74.7,74.2,73.7,71.0$ 70.4, 21.6. Anal. Calcd for $\mathrm{C}_{32} \mathrm{H}_{26} \mathrm{FeO}_{3} \mathrm{~S}: \mathrm{C}, 70.33 ; \mathrm{H}, 4.80 ; \mathrm{S}, 5.87$. Found: C, 70.53; H, 4.95; S, 5.89 .

Dione 8f. Red oil (30\%). TLC (20\% AcOEt/hexanes, $\left.R_{f}=0.32\right)$; IR (film, $\left.\mathrm{cm}^{-1}\right) 2919$ (m), 2850 (w), 1673 (s), 1621 (s), 1514 (m), 1446 (m), 810 (m), 732 (m); ${ }^{1} \mathrm{H}$ NMR (200 MHz, CDCl 3 ) $\delta$ $8.13(\mathrm{~s}, 1 \mathrm{H}), 7.92(\mathrm{~m}, 2 \mathrm{H}), 7.46(\mathrm{~m}, 2 \mathrm{H}), 7.20(\mathrm{q}, J=6.6 \mathrm{~Hz}, 4 \mathrm{H}), 5.18$ (app. t, $J=1.8 \mathrm{~Hz}, 2 \mathrm{H})$, 4.97 (app. t, $J=1.6 \mathrm{~Hz}, 2 \mathrm{H}$ ), 4.71 (app. t, $J=1.8 \mathrm{~Hz}, 2 \mathrm{H}$ ), 4.58 (app. t, $J=1.8 \mathrm{~Hz}, 2 \mathrm{H}$ ), 2.38 (s, 
3H). ${ }^{13} \mathrm{C}$ NMR $\left(75.5 \mathrm{MHz}, \mathrm{CDCl}_{3}\right) \delta 191.7,189.9,143.5,142.0,139.7,139.2,134.9,130.1$, 129.2, 127.3, 126.1, 125.2, 123.7, 123.0, 80.6, 80.4, 75.2, 74.4, 73.1, 71.2, 21.5. HRMS (EI) Calcd for $\mathrm{C}_{27} \mathrm{H}_{20} \mathrm{FeO}_{2} \mathrm{~S}_{2}$ 496.0254. Found 496.0260.

Dione 8c. Orange-brown oil (21\%). TLC (20\% AcOEt/hexanes, $\left.R_{f}=0.34\right)$; IR (film, cm $\left.{ }^{-1}\right) 2924$ (m), 2852 (w), 1674 (s), 1635 (s), 1368 (m), 810 (s); ${ }^{1} \mathrm{H}$ NMR (200 MHz, CDCl $\left.{ }_{3}\right) \delta 7.94(\mathrm{~d}, 2 \mathrm{H})$, 7.24 (bs, 4H), 6.97 (d, 2H), 5.00 (app. t, $J=1.6 \mathrm{~Hz}, 2 \mathrm{H}$ ), 4.92 (app. t, $J=1.6 \mathrm{~Hz}, 2 \mathrm{H}$ ), 4.63 (app. t, $J=1.6 \mathrm{~Hz}, 2 \mathrm{H}), 4.53$ (app. t, $J=1.8 \mathrm{~Hz}, 2 \mathrm{H}), 3.90$ (s, 3H), 2.40 (s, 3H). ${ }^{13} \mathrm{C}$ NMR $(75.5 \mathrm{MHz}$, $\left.\mathrm{CDCl}_{3}\right) \delta 196.4,191.7,163.1,139.8,135.1,132.1,130.9,130.1,123.9,113.8,80.7,80.4,74.7$, 74.2, 73.7, 71.0, 55.7, 21.6. HRMS (EI) Calcd for $\mathrm{C}_{26} \mathrm{H}_{22} \mathrm{FeO}_{3} \mathrm{~S}$ 470.0639. Found 470.0642.

Dione 8h. Dark red crystals (17\%). TLC (20\% AcOEt/hexanes, $\left.R_{f}=0.41\right)$; mp 123-124 ${ }^{\circ} \mathrm{C}$ (hexanes); IR (film, cm $\left.{ }^{-1}\right) 2923$ (m), 1674 (s), 1646 (s), 1530 (m), 1349 (m), 811 (m), 719 (m); ${ }^{1} \mathrm{H}$ NMR $\left(200 \mathrm{MHz}, \mathrm{CDCl}_{3}\right) \delta 8.84(\mathrm{bs}, 1 \mathrm{H}), 8.41(\mathrm{~m}, 1 \mathrm{H}), 8.21(\mathrm{~d}, J=7.8 \mathrm{~Hz}, 4 \mathrm{H}), 7.68(\mathrm{t}, J=$ $8.2 \mathrm{~Hz}, 1 \mathrm{H}$ ), 7.24 (bs, 4H), 4.97 (m, 4H), 4.75 (app. t, $J=1.8 \mathrm{~Hz}, 2 \mathrm{H}$ ), 4.60 (app. t, $J=2.0 \mathrm{~Hz}$, $2 \mathrm{H}), 2.40(\mathrm{~s}, 3 \mathrm{H}) .{ }^{13} \mathrm{C}$ NMR $\left(75.5 \mathrm{MHz}, \mathrm{CDCl}_{3}\right) \delta 195.7,191.3,148.1,140.5,140.0,135.0$, 134.3, 130.2, 130.0, 126.6, 123.5, 80.8, 79.0, 75.6, 74.1, 73.7, 71.2, 21.6. Anal. Calcd for $\mathrm{C}_{25} \mathrm{H}_{19} \mathrm{FeNO}_{4} \mathrm{~S}: \mathrm{C}, 61.87 ; \mathrm{H}, 3.95 ; \mathrm{N}, 2.89 ; \mathrm{S}, 6.61$. Found: C, 61.96; H, 4.15; N, 2.63; S, 6.72.

Dione 8i. Orange solid (11\%). TLC (20\% AcOEt/hexanes, $\left.R_{f}=0.47\right)$, mp $167-169{ }^{\circ} \mathrm{C}$ (hexanes); IR (film, cm ${ }^{-1}$ ) 2910 (m), 1672 (s), 1614 (s), 807 (s); ${ }^{1} \mathrm{H}$ NMR (200 MHz, $\left.\mathrm{CDCl}_{3}\right) \delta 7.38$ (d, 2H), $7.26(\mathrm{~d}, 2 \mathrm{H}), 5.10$ (bs, 2H), 4.99 (bs, 2H), 4.95 (bs, 2H), 4.61 (bs, 2H), 4.57 (bs, 2H), 4.51 (bs, $2 \mathrm{H}), 4.51(\mathrm{bs}, 2 \mathrm{H}), 4.20(\mathrm{~s}, 5 \mathrm{H}), 2.41(\mathrm{~s}, 3 \mathrm{H}) .{ }^{13} \mathrm{C} \mathrm{NMR}\left(75.5 \mathrm{MHz}, \mathrm{CDCl}_{3}\right) \delta 198.5,191.9$, 139.9, 135.1, 130.2, 124.0, 82.2, 80.2, 80.1, 74.6, 74.3, 72.5, 72.1, 70.8, 70.7, 70.3, 21.6. Anal. Calcd for $\mathrm{C}_{29} \mathrm{H}_{24} \mathrm{Fe}_{2} \mathrm{O}_{2} \mathrm{~S}: \mathrm{C}, 63.53 ; \mathrm{H}, 4.41 ; \mathrm{S}, 5.85$. Found: C, 63.23; H, 4.51; S, 5.99.

Dione 8I. Red oil (14\%). TLC (20\% AcOEt/hexanes, $\left.R_{f}=0.22\right)$; IR (film, cm $\left.{ }^{-1}\right) 2929(\mathrm{~m}), 28512$ (w), 1673 (s), 1634 (s), 811 (s), 771 (m); ${ }^{1} \mathrm{H}$ NMR (200 MHz, $\left.\mathrm{CDCl}_{3}\right) \delta 7.61$ (dd, $J=8.2,1.8 \mathrm{~Hz}$, 1H), 7.47 (d, $J=1.8 \mathrm{~Hz}, 1 \mathrm{H}$ ), 7.24 (bs, 4H), 6.91 (d, $J=8.4 \mathrm{~Hz}, 1 \mathrm{H}$ ), 5.01 (app. t, $J=2.2 \mathrm{~Hz}$, 2H), 4.93 (app. t, $J=2.2 \mathrm{~Hz}, 2 \mathrm{H}$ ), 4.64 (app. t, $J=2.2 \mathrm{~Hz}, 2 \mathrm{H}$ ), 4.54 (app. t, $J=1.8 \mathrm{~Hz}, 2 \mathrm{H}$ ), 3.97 (s, 3H), 3.94 (s, 3H), 2.40 (s, 3H). ${ }^{13} \mathrm{C}$ NMR $\left(75.5 \mathrm{MHz}, \mathrm{CDCl}_{3}\right) \delta$ 196.3, 191.6, 152.8, 149.1, 139.8, 135.0, 132.1, 130.1, 123.9, 123.2, 111.4, 110.1, 80.8, 80.4, 74.6, 74.2, 73.8, 71.0, 56.3, 21.6. HRMS (EI) Calcd for $\mathrm{C}_{27} \mathrm{H}_{24} \mathrm{FeO}_{4} \mathrm{~S} 500.0745$. Found 500.0748.

Dione 8j. Red oil (22\%). \%). TLC (20\% AcOEt/hexanes, $\left.R_{f}=0.32\right)$; IR (film, $\left.\mathrm{cm}^{-1}\right) 3056(\mathrm{w})$, 2920 (w), 1673 (s), 1642 (s), 1596 (m), 1492 (m), 867 (m), 810 (s); ${ }^{1} \mathrm{H}$ NMR (200 MHz, CDCl $\left.{ }_{3}\right)$ $\delta 8.42(\mathrm{~s}, 1 \mathrm{H}), 7.96(\mathrm{~m}, 4 \mathrm{H}), 7.60$ (m, 2H), 7.11 (bs, 4H), 5.07 (app. t, $J=1.8 \mathrm{~Hz}, 2 \mathrm{H}$ ), 4.94 (app. t, $J=1.8 \mathrm{~Hz}, 2 \mathrm{H}$ ), 4.69 (app. t, $J=1.8 \mathrm{~Hz}, 2 \mathrm{H}), 2.36$ (s, 3H). ${ }^{13} \mathrm{C} \mathrm{NMR}\left(75.5 \mathrm{MHz}, \mathrm{CDCl}_{3}\right) \delta$ 198.0, 191.6, 139.7, 136.7, 135.3, 135.0, 132.6, 130.1, 129.5, 128.5, 128.2, 128.0, 127.0, 125.1, 123.8, 80.5, 80.3, 75.0, 74.2, 73.9, 71.1, 21.5. HRMS (EI) Calcd for $\mathrm{C}_{29} \mathrm{H}_{22} \mathrm{FeO}_{2} \mathrm{~S} 490.0690$. Found 490.0682 . 


\section{Acknowledgements}

E. P.-C. wishes to thank Professor Cecilio Alvarez for performing HRMS analyses of some of the derivatives prepared. C. G. R.-C. wishes to thank both CONACyT and CONCyTEG (Mexico) for graduate scholarships. Support from the program "Fomento a la Investigación" of the University of Guanajuato is gratefully acknowledged.

\section{References}

1. E-mail L. S. L. chemLL1@emory.edu

2. For recent advances on ferrocene chemistry see the special issue of the Journal of Organometallic Chemistry on the $50^{\text {th }}$ Anniversary of the Discovery of Ferrocene $J$. Organomet. Chem. 2001, 637.

3. Ferrocenes: Homogeneous Catalysis, Organic Synthesis, Material Science; Togni, A.; Hayashi, T., Eds; VCH: New York, 1995.

4. (a) Top, S.; Vessières, A.; Leclerq, G.; Quivy, J.; Tang, J.; Vaisserman, J.; Huché, M.; Jaouen, G. Chem. Eur. J. 2003, 9, 5223. (b) Top, S.; Vessières, A.; Cabestaing, C.; Laïos, I.; Leclerq, G.; Provot, C.; Jaouen, G. J. Organomet. Chem. 2001, 637, 500.

5. (a) Carollo, L.; Curulli, A.; Floris, B. Appl. Organomet. Chem. 2003, 17, 589 (b) Sarhan, A. E-W.; Nouchi, Y.; Izumi, T. Tetrahedron 2003, 59, 6353.

6. Denifl, P.; Hradsky, A.; Bildstein, B.; Wurst, K. J. Organomet. Chem. 1996, 523, 79.

7. Miller, E. J.; Weigelt, C. A.; Serth, J. A.; Rusyid, R.; Brenner, J.; Luck, L. A.; Godlewski, M. J. J. Organomet. Chem. 1992, 440, 91.

8. (a) Wittenberg, R.; Srögl, J.; Egi, M.; Liebeskind, L. S. Org. Lett. 2003, 5(17), 3033. (b) Liebeskind, L. S.; Srögl, J. J. Am. Chem. Soc. 2000, 122, 11260. (c) Yu, Y.; Liebeskind, L. S. J. Org. Chem. 2004, 69 (10), 3554.

9. For other examples of cross-couplings of sulfur-containing substrates see: (a) AguilarAguilar, A.; Peña-Cabrera, E.; Liebeskind, L. S. ARKIVOC 2004, (i), 156. (b) Kusturin, C.; Liebeskind, L. S.; Rahman, H.; Sample, K.; Schweitzer, B.; Srogl, J.; Neumann, W. L. Org. Lett. 2003, 5(23), 4349. (c) Savarin, C.; Srogl, J.; Liebeskind, L. S. Org. Lett. 2001, 3(1), 91 (d) Savarin, C.; Liebeskind, L. S. Org. Lett 2001, 3(14), 2149. (e) Srogl, J.; Liebeskind, L. S. Org. Lett. 2002, 4(6), 979. (f) Kusturin, C.; Liebeskind, L. S.; Neumann, W. L. Org. Lett. 2002, 4(6), 983. (g) Liebeskind, L. S.; Srogl, J.; Savarin, C.; Polanco, C. Pure Appl. Chem. 2002, 74(1), 115. (h) Egi, M.; Liebeskind, L. S. Org. Lett. 2003, 5(6), 801. (i) Alphonse, F.A.; Suzenet, F.; Keromnes, A.; Lebret, B.; Guillaumet, G. Org. Lett. 2003, 5, 803.

10. (a) Suzuki, A. J. Organomet. Chem. 1999, 576, 147. (b) Stanforth, S. P. Tetrahedron 1998, 54, 263. (c) Tyrrell, E.; Brookes, P. Synthesis 2004, 469.

11. (a) Tokuyama, H.; Yokoshima, S.; Yamashita, T.; Fukuyama, T. Tetrahedron Lett. 1998, 39, 3189. (b) Neises, B.; Steglich, W. Angew. Chem., Int. Ed. 1978, 17(7), 522. 
12. Miyazaki, T.; Han-ya, Y.; Tokuyama, H.; Fukuyama, T. Synlett 2004, 477.

13. Taber, D. F.; Petty, E. H.; Raman, K. J. Am. Chem. Soc. 1985, 107,196.

14. 1,1'-Ferrocenedicarboxylic acid is commercially available.

15. For the generation of 1,1'-bislithioferrocene see McCulloch, Brubaker Jr.; C. H. Organometallics 1984, 3, 1707.

16. (a) Miyazaki, T.; Han-ya, Y.; Tokuyama, H.; Fukuyama, T. Synlett 2004, 477. (b) Tokuyama, H.; Miyazaki, T.; Yokoshima, S.; Fukuyama, T. Synlett 2004, 477. (c) Tokuyama, H.; Yokoshima, S.; Yamashita, T.; Fukuyama, T. Tetrahedron Lett. 1998, 39, 3189.

17. Zhang, S.; Zhang, D.; Liebeskind, L. S. J. Org. Chem. 1997, 62, 2312.

18. CAUTION! Care should be exercised at this point since it is well known that the complex $\mathrm{FcLi}_{2} \mathrm{TMEDA}$ is highly pyrophoric.

19. Skrydstrup, Hansen, S. G.; Laursen, B.; Rasmussen, B. S.; Larsen, J.; Sorensen, H. S.; T.; Amatore, C.; Jutand, A. Organometallics 2002, 21, 5243.

20. Meth-Cohn, O.; Walshe, N. D. A.; Bin Din, L. Tetrahedron Lett. 1979, 49, 4783.

21. Voguel, M.; Rosenberg, H.; Rausch, M. J. Org. Chem. 1957, 22, 903.

22. Carollo, L.; Curulli, A.; Floris, B. Appl. Organomet. Chem. 2003, 17, 589.

23. Sarhan, A.; Nouchi, Y.; Izumi, T. Tetrahedron. 2003, 6353. 